\title{
ATUAÇÃO FISIOTERAPÊUTICA NA NEUROPATIA DIABÉTICA: REVISÃO INTEGRATIVA
}

\author{
PHYSIOTHERAPETIC ACTUATION IN DIABETIC NEUROPATHY: INTEGRATIVE \\ REVIEW
}

\section{RESUMO}

Objetivo: Avaliar o efeito da atuação fisioterapêutica na neuropatia diabética. Método: Foi realizada uma revisão integrativa da literatura em redes eletrônicas: Medline, Lilacs, SciELO, PEDro e Google acadêmico, utilizando os seguintes descritores segundo o Decs/MeSH com suas respectivas traduções: "Neuropatia diabética" and "fisioterapia" and "reabilitação". Foram incluídos artigos publicados no intervalo temporal de 2008-2018 no idioma da língua portuguesa e inglesa. Não houve restrição quanto ao tipo de intervenção fisioterapêutica utilizada. Não foram inseridos estudo de revisão ou que não apresentassem intervenção fisioterapêutica. Resultados: Foram encontrados um total de 60 artigos, destes 42 foram excluídos, resultando em um montante de 17 para inserção na revisão e análise. Dentre os estudos elegíveis após a leitura do resumo, foram incluídos 09. Conclusão: Verificou-se que o fisioterapeuta pode atuar desde a prevenção até a reabilitação do paciente acometido pela doença através de vários métodos e técnicas para estimular a sensibilidade, trabalhar força, equilíbrio e propriocepção, também pode fazer orientações quanto ao cuidado com os pés e hábitos de vida saudável. No tocante à sensibilidade e dor muitos estudos mostraram melhora significativa.

Palavras-chave: Neuropatias diabéticas. Fisioterapia. Reabilitação.

\section{ABSTRACT}

Background: To evaluate the effect of physiotherapy on diabetes neuropathy. Method: An integrative review was performed on electronic network: Medline, Lilacs, SciELO, PEDro e Google scholar, using the followed Decs/MeSH keywords with their respective translations: "diabetics neuropathy" AND "physiotherapy" e "diabetics neuropathy" AND "rehabilitation". We included articles published in 2008-2018 time

1 Doutora em Evolução Humana: antropologia física e forense pela Universidade de Granada (Espanha). Docente do Centro Universitário de João Pessoa (UNIPÊ). E-mail: Iuciana_momaso@hotmail.com

2 Graduado em Fisioterapia pela UNINASSAU. E-mail: gerbesonmuniz@hotmail.com

3 Graduando em Fisioterapia pelo Centro Universitário de João Pessoa (UNIPÊ). E-mail: emerson.b.fritas@hotmail.com

4 Graduando em Fisioterapia pelo Centro Universitário de João Pessoa (UNIPÊ). E-mail: Cezar_Fellinto@outlook.com 
range in portuguese and english language. There was no restriction regarding the type of physical therapy interventions used. No review study or study that did not present physical therapy interventions were inserted. Results: 60 articles were found in total, 48 were excluded, resulting on 18 articles for insertion in the review and analysis. Among the eligible studies after reading the abstracts, 12 were included. Conclusion: It were found that the physiotherapist could act from prevention to rehabilitation of the patient affect by this disease throughout the various methods and techniques to stimulate the sensibility, gain of strength, balance and proprioception, can also provide guidance on foot care and health habits. Regarding the sensibility and pain, many studies shown a significant improvement.

Keywords: diabetic neuropathy; physiotherapy; rehabilitation.

\section{INTRODUÇÃO}

O Diabetes mellitus (DM) é uma perturbação crônica e complicada descrito por comprometimento do metabolismo da glicose e de outras substâncias produtoras de energia, em conjunto a uma variedade de complicações em órgãos eficazes para a sustentação da vida (PIRES; SANDOVAL, 2010). Uma das características é a ausência de insulina, que como decorrência, faz com que a glicose não seja aproveitada devidamente pelas células (CENCl et al., 2013).

Segundo Santos et al., (2015) o DM é apontado como um problema de saúde pública, haja em vista que ele afeta populações de países, em todas as fases do desenvolvimento. De acordo com Flor e Campos (2017), o DM consiste em principal causa de morbidade e mortalidade. Estima-se mundialmente que 382 milhões de pessoas se encontram com DM (8,3\%), esse número pode atingir 592 milhões em 2035; admite-se até então que cerca de 50,0\% dos diabéticos não sabem que estão com a doença, quanto à mortalidade avalia-se que 5,1 milhões de indivíduos com idade de 20 a 79 anos morreram devido ao diabetes em 2013.

Conforme dados da Organização Mundial de Saúde, o Brasil possui cerca de 10 milhões de diabéticos, é o $6^{\circ}$ país do mundo em número de pessoas com a doença (SANTOS et al., 2015). A incidência cresce consideravelmente como decorrência da relação genética e aquisição de fatores de risco que são relevantes para a patologia e dentre eles pode destacar: maior percentagem de urbanização, aumento da perspectiva de vida, industrialização, ingestão demasiado de dietas calóricas e ricas em hidratos de carbono de absorção rápida, variações do estilo de vida, sedentarismo, obesidade e maior sobrevida do paciente (SOUZA et al., 2013). Ela pode ser classificada em tipo 1 e 2, diabetes gestacional e outros tipos (SANTOS et al., 2015).

O diagnóstico antecipado da disfunção decorrente do DM, em conjunto ao tratamento apropriado e ao cuidado com os pés, aliado ao controle glicêmico, a 
instrução nutricional e cautela alimentar, uma rotina de vida saudável e orientações específicos como o uso de calçados adequado podem prevenir maiores problemas (MENDONÇA; MORAIS; MOURA, 2017; MANTOVANI et al., 2013).

Um problema crônico corriqueiro é a limitação de a mobilidade articular (ULHOA et al., 2011). O pé do paciente diabético é submetido a uma ordem de modificações neuropáticas. As transições atuam de forma sinérgica e carregam maior risco para desencadear lesões cutâneas de difícil restauração, fato que expõe o indivíduo ao risco de adquirir infecções graves. A neuropatia diabética afeta aspectos sensoriais motores e autonômicos que juntos tornam essa complicação mais nociva, o problema, mas dominante nessa patologia é a neuropatia diabética periférica porque é constante e irreversível (OLIVEIRA; MARCHI; LEGUISAMO, 2016; SALES; SOUZA; CARDOSO, 2012).

A neuropatia afeta o sistema nervoso periférico (sensitivo, motor e autonômico), capaz de causar alterações de sensibilidade vibratória, cinestesia e sensibilidade tátil, seguindo para uma redução na propriocepção, fraqueza muscular; déficit de pressão e falta de equilíbrio, que resulta no surgimento de úlceras de pressão na planta dos pés com risco de quedas e amputações nos membros inferiores (FREGONESI; CAMARGO, 2010; CENCl et al., 2013).

A tática de manuseio ajuda na prevenção à descoberta precoce e a intervenção correta das alterações do DM, que diminui o impacto socioeconômico, não só para o paciente diabético, mas também para a população, assim diminuindo a morbidade (ULHOA et al., 2011). A fisioterapia realiza a avaliação de risco do surgimento da neuropatia diabética e na estimulação da regeneração da fibra nervosa (BARRILE et al., 2013).

Segundo Carvalho et al., (2009) a neuropatia diabética induz a redução ou detrimento da sensibilidade protetora dos pés, que deixa o diabético mais sensível a lesões ou trauma mecânico, por conseguinte, leva ao desenvolvimento de danos cutâneo e provavelmente, perda segmentar nos membros inferiores. A neuropatia periférica é uma condição clínica que afeta o sistema nervoso periférico, ocasionando dormência, formigamento e dor nos membros (FORTE et al., 2016)

Durante os atendimentos práticos da disciplina de neurologia foi possível perceber a importância da atuação do fisioterapeuta no tratamento da neuropatia diabética. Sendo possível observar que pacientes podem apresentar déficit de sensibilidade em MMIl e alterações de equilíbrio devido a doença, através desse achado cinético funcional de início foi observado um paciente que apresentava todos esses déficits e alterações. Dando início a toda a pesquisa. Assim, a concepção é que o trabalho proposto poderá auxiliar aos professores da área, bem como aos discentes e qualquer um que se interesse pelo tema abordado, podendo servir como fonte de pesquisa. Diante do exposto surgiu a seguinte pergunta de investigação: qual o efeito da fisioterapia nas disfunções sensoriais e motoras da neuropatia diabética? Sendo assim o objetivo desse trabalho é realizar 
uma revisão de literatura interativa para avaliar o efeito da atuação fisioterapêutica na neuropatia diabética.

\section{REVISÃO BIBLIOGRÁFICA}

\section{DIABETES MELLITUS E A NEUROPATIA DIABÉTICA}

O Diabetes Mellitus (DM) significa uma enfermidade crônica, caracterizada pela alteração dos indicadores glicêmicos, seguida por uma série de complicações, sobretudo quando diagnosticado de forma tardia (SILVA et al., 2012). Também caracterizado por falhas na secreção e atuação da insulina (SOUZA et al., 2013). O DM é estimado como uma doença de etiologia múltipla, consequente da ausência de insulina ou incompetência da mesma desempenhar normalmente suas funções e efeitos. Tem como consequência a hiperglicemia crônica que apresentam distúrbios do metabolismo, carboidratos, lipídios e proteína (CARDOSO et al., 2011).

O Diabetes Mellitus por sua vez se classifica em: Tipo 1, Tipo 2, gestacional e outros tipos específicos. O DM Tipo Imanifesto como diabetes do jovem está presente em $5 \%$ a $10 \%$ dos casos de diabetes, distinguir-se por extermínio das células beta do pâncreas (comumente ocasionada por procedimento autoimune), como consequência a perda na produção e também secreção da insulina. O DM tipo 2 prevalece em $90 \%$ a $95 \%$ dos casos de DM e distinguir-se pela resistência à atuação e a ausência da insulina revelar-se pela inabilidade de contrabalançar essa resistência. O DM2 constitui um grave problema de saúde pública pelo alto predomínio no mundo que é maior entre os idosos, pela morbidade e por ser um dos fundamentais fatores de risco cardiovascular e cerebrovascular (PETERMANN et al., 2015; PASQUALOTO; ALBERTON; FRIGERI, 2012; MENDES et al., 2011).

O DMG é determinado com algum nível de diminuição da tolerância á glicose, onde o princípio ou descoberta acontece durante a gravidez, podendo ou não permanecer após o parto. No andamento pós-gestacional há diminuição da concentração plasmática contra insulínicos, decaindo as necessidades maternas de insulina e a glicose retornando à normalidade. No entanto as grávidas que apresentam DMG em seguida têm elevado risco de desenvolverem DM2 (SILVA et al., 2017; FERREIRA et al., 2011).

O DM tem relação com várias comorbidades que afetam ainda mais a funcionalidade do paciente, a restrição na habilidade funcional diminui também a convivência social e senso de bem-estar (ANJOS et al., 2012). Há maior dificuldade de saúde em portadores de DM são as úlceras nos membros inferiores na região dos pés (CISNEROS, 2010). O tratamento de início constitui em regular os níveis de glicose no sangue, com terapia medicamentosa com atividades físicas ou agregação de ambos (FORTE et al., 2016). 
São desencadeados desequilíbrios no sistema tônico-postural, gerando forças compensatórias atípicas, levando a mudanças nos parâmetros cinéticos do centro de pressão plantar, tanto em condições estáticas, quanto dinâmicas (NOZABIELl et al., 2012). Salienta que o entendimento sobre a enfermidade é eficaz para a manipulação adequada da condição crônica, mas apenas isso não se exprime em mudar de procedimento. Isso quando o conhecimento é influenciado por atributos individuais, por ser constituído que o indivíduo comina á condição crônica e por seus costumes diante dela (TESTON et al., 2017).

Com isso passamos a entender que o pé diabético é o conjunto das alterações sensório-motoras, vasculares (micro e macrovasculares) e biomecânicas que levam à deformidade em decorrência da neuropatia. O diagnóstico do pé diabético depende do exame clinico adequado, ou seja, uma boa anamnese e exame físico. É uma complicação crônica que ocorre em média após dez anos de evolução da doença (BORGES; CARDOSO, 2010).

Segundo Cisneros (2010), o consenso internacional sobre pé diabético, avigorando os relatos de estudos distintos sobre amputação desses pacientes, recomenda ações multiprofissionais para se alcançar a diminuição de $50 \%$ nas amputações almejada na European Declaration of St Vicent: inspeção dos pés dessas pacientes durante as visitas clínica, uso de calçados adaptados, educação para o autocuidado e monitoramento continuo daqueles que já mostrar-se com lesões nos pés. Para o auxílio de portadores de diabetes com risco de lesão neuropática por insensibilidade. Essas ações são apropriadas para redução a incidência e a ocorrência de lesões neuropáticas nesses indivíduos.

Conforme Picon (2011), a insulina é definida como um nerve growth factors (NGF fator de crescimento nervoso) e, contudo é capaz de gerar importante apoio neurotrófico aos neurônios sensitivos, motores e autonômicos, há uma teoria de que com a decadência da agilidade da insulina, o diabético diminui o auxílio neurotrófico e consente que a neuropatia gradativamente se instale.

Conforme Barrile et al. (2013), a neuropatia é uma grave alteração crônica do DM, consisti em um distúrbio no nervo periférico trajado por atrofia e degradação axonal ou por adulteração nas células de Schwann, gerando desmielinização em partes dos axônios, tendo como decorrência redução da rapidez de deslocamento ou suspensão da transmissão do impulso nervoso. As úlceras aparentes ou danos pré-ulcerativas nos pés de pacientes diabéticos com sensibilidade diminuída acarretada por neuropatia diabética constitui $85 \%$ das ocorrências graves de internamentos hospitalares. Com a diminuição de a sensibilidade plantar e das informações provenientes dos mecanorreceptores, há também restrição sobre o equilíbrio (ANJOS et al., 2012).

Segundo a Sociedade Brasileira de Diabetes, a neuropatia diabética pode ser dividida em: neuropatia sensitiva, que causa lesão progressiva da sensibilidade dolorosa, do discernimento de pressão, temperatura e propriocepção; neuropatia 
motora, que ocasiona atrofiae fraqueza dos músculosintrínsecosdospés, procedendo em defeitos e protótipo anormal da marcha; neuropatia autonômica, que leva a ausência ou diminuição da secreção sudorípara, causando ressecamento da pele, com aparecimento de rachaduras e fissuras (PEDROSA, 2009).

Atos preventivos são básicos para atenuar complicações avassaladoras da neuropatia diabética (SARTOR, 2014). As neuropatias doloridas são regulares e quase sempre de difícil tratamento (KRAYCHETE; SOKATA, 2011). A qual é distinguida pela redução da velocidade de condução nervosa, que está integrada a uma diminuição da atividade da bomba de sódio-potássio no nervo. Essa alteração atenta distúrbios metabólicos e isquêmicos (BORGES; CARDOSO, 2010).

\section{ATUAÇÃO FISIOTERAPÊUTICA NA NEUROPATIA DIABÉTICA}

Segundo Portes (2015), o Ministério da Saúde redefiniu em 2014 a Rede de Atenção à Saúde das Pessoas com Doenças Crônicas no âmbito do Sistema Único de Saúde, ressaltando a seriedade das equipes multiprofissionais para o cuidado dos usuários. Dentre as profissões que compõem as equipes, vale destacar a fisioterapia, uma ciência sobreposta que tem como artifício de estudo o movimento humano nas suas deformações patológicas e repercussões psíquicas e orgânicas. Aponta preservar, manter, desenvolver ou restaurar a integridade de órgão, sistema ou função.

O fisioterapeuta é o profissional do campo da saúde que oferece serviços a pessoas e populações com finalidade de desenvolver, manter e restaurar o movimento e a capacidade funcional da pessoa. Em meio as suas capacidades, atua na prevenção da saúde, promoção, reabilitação ou cura de portadores de disfunções orgânicas, agudas ou crônicas, que podem afetar a aptidão de concretizar trabalho físico, lazer ou autocuidado. Pode também atuar em conjunto com equipe multidisciplinar no tratamento e na prevenção da Neuropatia do Diabetes Mellitus (FURIERI, 2015).

Torna-se imprescindível para a prática clínica da fisioterapia a identificação de informações sensoriais proveniente de receptores cutâneos plantares e a manutenção da força muscular nos diabéticos, de forma preventiva, a fim de preservar a funcionalidade e evitar quedas nesses indivíduos (VERAS et al., 2015).

São vários os métodos de avaliação do risco de ulceração do pé diabético mencionados por diversos autores, sendo uniformes em declarar efetiva a identificação do pé em risco de ulceração. É formidável realizar a avaliação dos pés de forma criteriosa, fazendo parte do exame físico (SILVA et al., 2014).

O monofilamento SW é um dos testes mais utilizados para a avaliação da sensibilidade cutânea, estimado padrão ouro para medidas de sensibilidade das fibras finas de adequação lenta e de lesões dos nervos periféricos. Desempenham sobre a pele pesos equivalentes a 0,$05 ; 0,2 ; 2,0 ; 4,0 ; 10,0$ e 300,0 gramas, analisando 
a diminuição protetora a partir do monofilamento de 2,0 gramas, adjunto a mais um teste de sensibilidade: vibratória (uso do diapasão de $128 \mathrm{~Hz}$ ); sensibilidade tátil (uso de algodão) ou a investigação de reflexos plantares e calcâneos. Não há consenso específicos quanto ao local a ser testados e o número mínimo de pontos não sentidos necessários para o prognóstico ideal de pé ulceração (SILVA, 2015; SILVA et al., 2014).

O fisioterapeuta pode colaborar na prevenção e recuperação das alterações sensoriais e motoras em pessoas acometidas por neuropatia diabética, consequentemente estudos como este são formidáveis para expandir os conhecimentos a respeito da doença e suas decorrências (SILVA; RODRIGUES; HONÓRIO, 2009). A prevenção deve ser a primeira série de combate às úlceras diabéticas. Sabendo que para uma prevenção apropriada das úlceras plantares e das amputações é necessário identificar o risco (CUBA et al., 2013).

Conforme o estudo de Fortaleza et al. (2010) mostra a seriedade de um manejo preventivo em pessoas portadoras do diabetes, para que possam ser orientados quanto aos cuidados gerais para prevenção de inabilidade podendo, assim, diminuir a probabilidade de desenvolver problemas vindouros. De acordo com Silva (2015) se faz necessário recomendar uma intervenção que auxilie na promoção da saúde desses indivíduos atuando na prevenção de alterações advindas da patologia, para que possam desenvolver de modo autônomo as suas atividades de vida diária.

A atuação da fisioterapia ante o portador de diabetes deve abranger desde a prevenção de ulceração até o controle e reabilitação. O objetivo e o procedimento devem estar fundamentados na apresentação clínica individual de cada indivíduo por isso a necessidade de se fazer uma avaliação detalhada (FURIERI, 2015). Barros et al. (2017) afirmam que a intervenção fisioterapêutica, além dos efeitos diretos que podem ser esperados da aplicação isolada ou em conjunto e da ampla gama de alternativas terapêuticas a seu dispor, podendo colaborar com ações de educação em saúde, sendo uma ferramenta importante para conscientizar os integrantes a modificar seus hábitos em relação ao autocuidado e atitudes que possam colocar em risco de lesões nos pés de pessoas diabéticas.

De acordo com Dantas et al.(2014), as condutas fisioterapêuticas em pessoas com sequelas do tipo pé diabético são de suma importância, tanto no suporte, a prevenção quanto ao estabelecimento de metas e estratégias de cuidado.

O fisioterapeuta realiza um trabalho essencial na reabilitação do paciente acometido pela neuropatia diabética, e um início precoce do tratamento adequado influência nos resultados ocasionais da reabilitação. O fisioterapeuta deverá preocupar-se com as condições do coto e a qualidade físicas do paciente, para evitar complicações (PIRES; SANDOVAL, 2010). 


\section{METODOLOGIA}

Foi realizada uma revisão integrativa da literatura nas bases de dados: MEDLINE, Lilacs, SciELO, PEDro e Google acadêmico, utilizando os seguintes descritores Decs/MeSH com suas respectivas traduções para o português: "diabetics neuropathy" AND "physiotherapy" e "diabetics neuropathy" AND "rehabilitation".

Foram incluídos artigos publicados no intervalo temporal de 2008-2018 no idioma da língua portuguesa e inglesa. Não houve restrição quanto ao tipo de intervenção fisioterapêutica utilizada. Não foram inseridos estudo de revisão ou que não apresentassem intervenção fisioterapêutica.

Para execução da revisão integrativa seguiu-se os seguintes passos: a) elaboração das perguntas nortea doras: qual o real efeito da atuação fisioterapêutica na atenção com o portador da neuropatia diabética? Qual o perfil da intervenção, modalidade e frequência terapêutica proposta para atuar diante da neuropatia diabética?; b) busca da amostragem nas bases de dados supracitadas; c) seleção e análise crítica dos dados, onde foram selecionadas as principais informações tais como: definição dos sujeitos, metodologia, tamanho da amostra, mensuração de variáveis, método de análise e conceito base empregados; d) apresentação dos resultados e discussão.

Foram encontrados um total de 60 artigos, destes 42 foram excluídos, resultando em um montante de 17 para inserção na revisão após análise. No entanto, ao final, somente 10 artigos foram inseridos no estudo (Figura 1).

Figura 1. Organograma da seleção dos artigos utilizados na revisão integrativa.

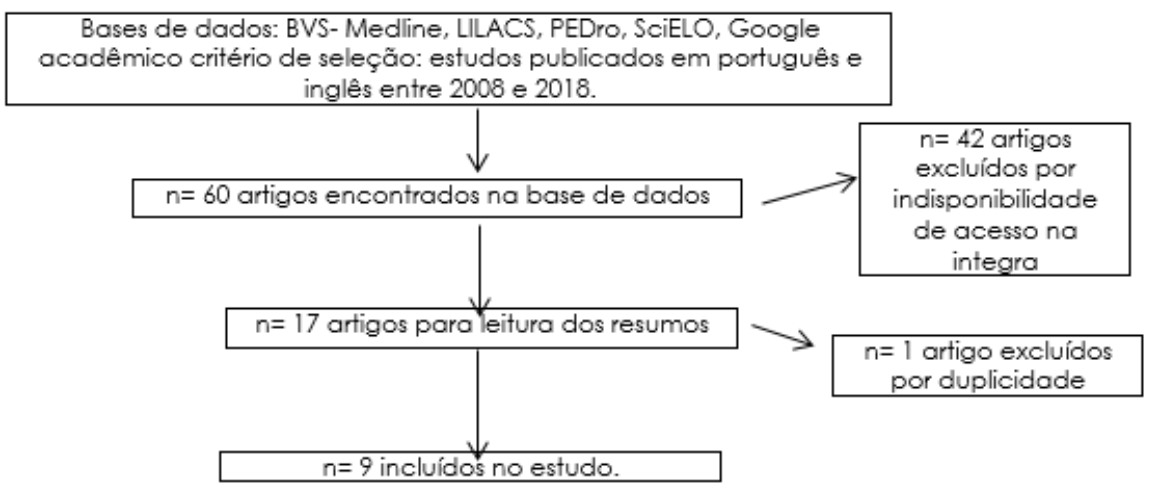

Fonte: Dados da pesquisa, 2019.

A plataforma Physiotherapy Evidence Database Scale (PEDro) é considerado um dos bancos de dados mais abrangentes para indexar relatos de ECR que investigam os efeitos de intervenções fisioterapêuticas (SHIWA et al., 2011), no entanto, além disso, utiliza e disponibiliza para avalição da qualidade dos artigos critérios baseados na escala Delphi desenvolvida por Verhagen e colaborares. 
Considerando os 11 pontos da escala Pedro, somente foram incluídos os artigos que recebiam pelo menos cinco pontos, sendo incluídos somente 9 (Quadro 1).

Quadro 1. Avaliação da qualidade dos artigos através.

\begin{tabular}{|c|c|c|c|c|c|c|c|c|c|}
\hline Itens & $\begin{array}{l}\text { Lima, } \\
\text { Junqué- } \\
\text { ra, } 2017\end{array}$ & $\begin{array}{l}\text { Piove- } \\
\text { san, } \\
2015\end{array}$ & $\begin{array}{l}\text { Cou- } \\
\text { tinho } \\
\text { et al.' } \\
2009\end{array}$ & $\begin{array}{l}\text { Barros } \\
\text { etal.' } \\
2017\end{array}$ & $\begin{array}{l}\text { Souza, } \\
2015\end{array}$ & $\begin{array}{l}\text { Magno } \\
\text { etal.' } \\
2017\end{array}$ & $\begin{array}{l}\text { Lee; } \\
\text { Lee; } \\
\text { Song, } \\
2013\end{array}$ & $\begin{array}{l}\text { Sartor } \\
\text { et al., } \\
2015\end{array}$ & $\begin{array}{l}\text { Santos } \\
\text { et al., } \\
2008\end{array}$ \\
\hline $\begin{array}{l}\text { Os critérios de elegibilidade } \\
\text { foram especificados }\end{array}$ & Sim & Sim & Não & Sim & Sim & Sim & Sim & Sim & Sim \\
\hline $\begin{array}{c}\text { Os sujeitos foram } \\
\text { aleatoriamente distribuídos } \\
\text { por grupos }\end{array}$ & Não & Sim & Sim & Sim & Sim & Sim & Sim & Não & Não \\
\hline $\begin{array}{l}\text { A distribuição dos sujeitos } \\
\text { foi cega }\end{array}$ & Não & Não & Não & Não & Não & Não & Não & Não & Não \\
\hline $\begin{array}{l}\text { Inicialmente, os grupos } \\
\text { eram semelhantes no que } \\
\text { diz respeito aos indicadores } \\
\text { de prognósticos mais } \\
\text { importantes }\end{array}$ & Não & Sim & Sim & Não & Sim & Sim & Sim & Sim & Sim \\
\hline $\begin{array}{l}\text { Todos os sujeitos } \\
\text { participaram de forma } \\
\text { cega no estudo }\end{array}$ & Não & Não & Não & Não & Não & Não & Não & Não & Não \\
\hline $\begin{array}{l}\text { Todos os fisioterapeutas } \\
\text { que administraram a } \\
\text { terapia fizeram-no de } \\
\text { forma cega }\end{array}$ & Não & Não & Não & Não & Não & Não & Não & Sim & Não \\
\hline $\begin{array}{l}\text { Todos os avaliadores que } \\
\text { mediram pelo menos um } \\
\text { resultado-chave, fizeram- } \\
\text { no de forma cega }\end{array}$ & Não & Não & Não & Não & Não & Não & Sim & Sim & Não \\
\hline $\begin{array}{l}\text { Medições de pelo menos } \\
\text { um resultado-chave foram } \\
\text { obtidas em mais de } 85 \% \\
\text { dos sujeitos inicialmente } \\
\text { distribuídos pelos grupos }\end{array}$ & Sim & Não & Sim & Sim & Sim & Sim & Sim & Sim & Sim \\
\hline $\begin{array}{c}\text { Todos os sujeitos a partir } \\
\text { dos quais se apresentaram } \\
\text { mediços de resultados } \\
\text { receberam o tratamento } \\
\text { ou a condição de controle } \\
\text { conforme a distribuição } \\
\text { ou, quando não foi esse } \\
\text { o caso, fez-se a anállise } \\
\text { dos dados para pelo } \\
\text { menos um dos resultados- } \\
\text { chave por "intenção de } \\
\text { tratamento" }\end{array}$ & Sim & Sim & Sim & Sim & Sim & Sim & Não & Sim & Sim \\
\hline $\begin{array}{c}\text { Os resultados das } \\
\text { comparaçoses estatísticas } \\
\text { intergrupos foram descritos } \\
\text { para pelo menos um } \\
\text { resultado-chave }\end{array}$ & Sim & Sim & Sim & Sim & Sim & Sim & Sim & Sim & Sim \\
\hline $\begin{array}{l}\text { O estudo apresenta tanto } \\
\text { medidas de precisãa como } \\
\text { medidas de variabilidade } \\
\text { para pelo menos um } \\
\text { resultado-chave }\end{array}$ & Sim & Sim & Sim & Sim & Sim & Sim & Sim & Sim & Sim \\
\hline Total & 05 & 06 & 06 & 06 & 07 & 07 & 07 & 08 & 06 \\
\hline
\end{tabular}

\section{RESULTADOS}

No que concerne ao perfil de apresentação dos artigos, a maioria foi publicada no ano de 2017 (n=3), em língua portuguesa (n=7), na base de dados SciELO ( $n=4)$ e com característica metodológica de ensaio clínico (n=8). No Quadro 
2 a distribuição dos autores/ano, os objetivos dos estudos, a amostra, a metodologia e os resultados obtidos.

Quadro 2. Distribuição dos autores/ano, os objetivos principais dos estudos, a amostra, a metodologia e os resultados obtidos.

\begin{tabular}{|c|c|c|c|c|}
\hline $\begin{array}{l}\text { AUTOR/ } \\
\text { ANO }\end{array}$ & OBJETIVO & AMOSTRA & MÉTODO & RESULTADOS \\
\hline LJ (2017) & $\begin{array}{l}\text { Avaliar o efeito de } \\
\text { uma intervenção } \\
\text { fisioterapêutica } \\
\text { no equilíbrio, } \\
\text { propriocepção e } \\
\text { coordenação de } \\
\text { pacientes diabéticos. }\end{array}$ & $\begin{array}{l}\text { A intervenção } \\
\text { foi realizada } \\
\text { com } 11 \text { sujeitos } \\
\text { com idade de } \\
61 \text { a } 77 \text { anos } \\
\text { portadores de } \\
\text { DM com perda } \\
\text { de sensibilidade, } \\
\text { não portadores } \\
\text { de NPD. }\end{array}$ & $\begin{array}{l}\text { Ensaio } \\
\text { clínico }\end{array}$ & $\begin{array}{l}\text { Observaram melhoras } \\
\text { significativas para a } \\
\text { goniometria de flexão plantar } \\
\text { direita e esquerda. Já na } \\
\text { percepção da sensibilidade } \\
\text { plantar, houve melhora da } \\
\text { sensibilidade dos pés. }\end{array}$ \\
\hline $\begin{array}{c}P \\
(2015)\end{array}$ & $\begin{array}{c}\text { Analisar os efeitos } \\
\text { de uma intervenção } \\
\text { fisioterapêutica em } \\
\text { idosas diabéticas tipo } \\
2 .\end{array}$ & $\begin{array}{l}\text { Foram avaliadas } \\
21 \text { pacientes } \\
\text { idosas com } \\
\text { diabetes tipo } 2 \\
\text { com idade de } 66 \\
\text { anos, divididas } \\
\text { em dois GE= } 11 \text { e } \\
\text { GC }=10 .\end{array}$ & $\begin{array}{l}\text { Ensaio } \\
\text { clínico }\end{array}$ & $\begin{array}{c}\text { Para ambos os pés, foi } \\
\text { encontrada diferença } \\
\text { estatística na distribuição } \\
\text { das cargas de pressão } \\
\text { plantar intragrupo no GE. } \\
\text { Encontrou-se diferença entre } \\
\text { os grupos pré-intervenção. } \\
\text { Na sensibilidade plantar } \\
\text { houve diferença significativa } \\
\text { intragrupo e entre grupos. } \\
\text { Para a dor em MMll, obteve } \\
\text { diferença. }\end{array}$ \\
\hline $\begin{array}{c}C \\
(2009)\end{array}$ & $\begin{array}{l}\text { Aferir a integridade do } \\
\text { músculo tibial anterior } \\
\text { das pacientes idosas } \\
\text { portadoras de DM, } \\
\text { através dos testes } \\
\text { de eletrodiagnóstico } \\
\text { e dinamometria, } \\
\text { analisando padrão } \\
\text { de força muscular, } \\
\text { coeficiente alfa e } \\
\text { tempo de resposta do } \\
\text { músculo ao estímulo } \\
\text { elétrico farádico nos } \\
\text { grupos, diabético e } \\
\text { hígido, antes e após } \\
\text { intervenção }\end{array}$ & $\begin{array}{l}\text { Pesquisa com } \\
16 \text { indivíduos } \\
\text { divididos em: } 8 \\
\text { diabéticos e } 8 \\
\text { hígidos. }\end{array}$ & $\begin{array}{l}\text { Estudo de } \\
\text { coorte }\end{array}$ & $\begin{array}{c}\text { No GDI as análises de } \\
\text { reobase e acomodação } \\
\text { variaram não apresentando } \\
\text { grau de significância. No } \\
\text { grupo diabético membro } \\
\text { intervenção e não } \\
\text { intervenção nas análises } \\
\text { em média de reobase, } \\
\text { acomodação e cronaxia } \\
\text { variaram não apresentando } \\
\text { grau de significância. Análise } \\
\text { dinamométrica entre o grupo } \\
\text { hígido e GDI mostraram } \\
\text { melhora em ambos, porém } \\
\text { com grau de significância } \\
\text { maior no GDI. }\end{array}$ \\
\hline$(2017)$ & $\begin{array}{l}\text { Avaliar o impacto } \\
\text { de uma intervenção } \\
\text { fisioterapêutica na } \\
\text { prevenção do pé } \\
\text { diabético. }\end{array}$ & $\begin{array}{l}\text { Pesquisa } \\
\text { realizada com } 24 \\
\text { usuários divididos } \\
\text { em três grupos de } \\
8 \text { indivíduos cada } \\
\text { com duração de } \\
8 \text { sessões. } \\
\end{array}$ & $\begin{array}{l}\text { Ensaio } \\
\text { clínico }\end{array}$ & $\begin{array}{c}\text { Após a intervenção } \\
\text { ocorreram alterações } \\
\text { estatisticamente significantes } \\
\text { em relação ao hábito de } \\
\text { cuidados dos pés. }\end{array}$ \\
\hline$\frac{S}{(2015)}$ & $\begin{array}{c}\text { Avaliar se um } \\
\text { programa } \\
\text { fisioterapêutico } \\
\text { traria mudanças na } \\
\text { qualidade de vida, na } \\
\text { mobilidade funcional, } \\
\text { na propriocepção, } \\
\text { na sensibilidade tátil e } \\
\text { dolorosa. }\end{array}$ & $\begin{array}{l}\text { Pesquisa com } \\
43 \text { idosos com } \\
\text { diagnostico de } \\
\text { DM, residentes } \\
\text { de cinco ILPI na } \\
\text { cidade de Porto } \\
\text { Alegre e região } \\
\text { metropolitana. }\end{array}$ & $\begin{array}{l}\text { Ensaio } \\
\text { clínico }\end{array}$ & $\begin{array}{c}\text { Após a intervenção, os idosos } \\
\text { do Gl apresentaram melhora } \\
\text { significativa do Escore Geral } \\
\text { do SF-36 e do Estado Geral } \\
\text { de Saúde e apresentam } \\
\text { escores significativamente } \\
\text { mais altos do que os idosos } \\
\text { do GC em termos de } \\
\text { Capacidade Funcional, } \\
\text { Aspectos Emocionais e } \\
\text { Escore Geral. }\end{array}$ \\
\hline
\end{tabular}

INTER/SCIENTIA

REVISTA INTERSCIENTIA | V. 7 | N. 2 | P. 109-130 | JUL-DEZ/2019 


\begin{tabular}{|c|c|c|c|c|}
\hline $\begin{array}{c}M \\
(2017)\end{array}$ & $\begin{array}{l}\text { Avaliar sensibilidade, } \\
\text { equilíbrio e qualidade } \\
\text { de vida em diabéticos } \\
\text { neuropatas, } \\
\text { comparando os } \\
\text { efeitos de dois } \\
\text { protocolos de } \\
\text { Fisioterapia. }\end{array}$ & $\begin{array}{c}\text { Intervenção foi } \\
\text { composta por } 10 \\
\text { diabéticos com } \\
\text { manifestações } \\
\text { neurológica } \\
\text { periférica, } \\
\text { divididos em } \\
\text { dois grupos GB e } \\
\text { GC ambos com } \\
\text { cinco voluntários } \\
\text { cada. }\end{array}$ & $\begin{array}{l}\text { Ensaio } \\
\text { clínico }\end{array}$ & $\begin{array}{c}\text { Referente a sensibilidade } \\
\text { houve acréscimo sobre os } \\
\text { pontos analisados, assim } \\
\text { como a estabilidade estática } \\
\text { e dinâmica, com reflexão } \\
\text { na QV dos participantes } \\
\text { em ambos os grupos. Cabe } \\
\text { ressaltar que no GC apenas } \\
\text { a EEB teve crescimento } \\
\text { considerável, enquanto } \\
\text { no GB todos os resultados } \\
\text { obtiveram significância } \\
\text { estatística, o que resultou } \\
\text { em melhoras no equilíbrio, } \\
\text { sensibilidade e refletiu na QV } \\
\text { dos voluntários. }\end{array}$ \\
\hline LLS (2013) & $\begin{array}{c}\text { Investigar os efeitos } \\
\text { da (WBV) combinada } \\
\text { com um programa } \\
\text { de exercícios de } \\
\text { equilíbrio no equilíbrio, } \\
\text { força muscular } \\
\text { e hemoglobina } \\
\text { glicosilada (HbAlc) } \\
\text { em pacientes idosos } \\
\text { com (NPD). }\end{array}$ & $\begin{array}{l}\text { A intervenção } \\
\text { foi composta } \\
\text { por } 55 \text { pacientes } \\
\text { diabéticos com } \\
\text { manifestações } \\
\text { neurológicas } \\
\text { periférica, } \\
\text { dividida em } 3 \\
\text { grupos GWBV, } \\
\text { GBE, GC. }\end{array}$ & $\begin{array}{l}\text { Ensaio } \\
\text { clínico }\end{array}$ & $\begin{array}{c}\text { O estudo observou que } \\
\text { houve Melhorias significativas } \\
\text { no equilíbrio estático, } \\
\text { equilíbrio dinâmico, força } \\
\text { muscular e HbAlc no grupo } \\
\text { WBV combinado com o } \\
\text { programa de exercícios de } \\
\text { equilíbrio, em comparação } \\
\text { com os grupos BE e controle. }\end{array}$ \\
\hline $\begin{array}{c}S \\
(2015)\end{array}$ & $\begin{array}{l}\text { Investigar os efeitos } \\
\text { do fortalecimento, } \\
\text { alongamento e } \\
\text { treinamento funcional } \\
\text { no processo de } \\
\text { rolagem do pé } \\
\text { durante a marcha. }\end{array}$ & $\begin{array}{c}\text { Pesquisa } \\
\text { randomizada } \\
\text { com } 55 \text { pacientes } \\
\text { divididos em GC: } \\
29 \text { e Gl: } 26 \text { por } 12 \\
\text { semanas. }\end{array}$ & $\begin{array}{l}\text { Ensaio } \\
\text { clínico }\end{array}$ & $\begin{array}{l}\text { A velocidade da passada } \\
\text { não foi estatisticamente } \\
\text { diferente entre grupos e } \\
\text { não diferiu na avaliação de } \\
\text { acompanhamento. }\end{array}$ \\
\hline $\begin{array}{c}S \\
(2008)\end{array}$ & $\begin{array}{l}\text { Avaliar a amplitude } \\
\text { da oscilação do } \\
\text { centro de pressão } \\
\text { na posição bipodal } \\
\text { com olhos abertos e } \\
\text { sensibilidade. }\end{array}$ & $\begin{array}{c}\text { Participaram } \\
\text { da intervenção } \\
13 \text { mulheres } \\
\text { diabéticas com } \\
\text { idade de } 50 \text { à } 70 \\
\text { anos. }\end{array}$ & $\begin{array}{l}\text { Ensaio } \\
\text { clínico }\end{array}$ & $\begin{array}{c}\text { Comparando a sensibilidade } \\
\text { antes, após seis e } 12 \\
\text { semanas de treinamento } \\
\text { proprioceptivo, notou-se } \\
\text { significativa melhora da } \\
\text { sensibilidade tátil plantar. } \\
\text { Em relação aos valores } \\
\text { médios referentes à } \\
\text { oscilação AP do centro de } \\
\text { pressão, houve diferença } \\
\text { significativa entre os valores } \\
\text { ao longo do tempo, antes da } \\
\text { intervenção. }\end{array}$ \\
\hline
\end{tabular}

Legenda: NPD: neuropatia diabética; GE: grupo experimental; MMII: membros inferiores; GDI: grupo diabético membro intervenção; DM: diabetes mellitus; Gl: grupo intervenção; GC: grupo controle; QV: qualidade de vida; EEB: escala de equilíbrio de Berg; WBV: vibração de corpo inteiro.

Fonte: Dados da pesquisa, 2019.

Observou-se que a maioria dos estudos analisados apresentaram resultados positivos quanto à atuação fisioterapêutica junto à neuropatia diabética, no entanto, no tocante ao tipo de intervenção, tempo do programa de intervenção e de sessão, assim como a frequência e variáveis estudadas, o Quadro 3 a seguir, os apresenta resumidamente. 
Quadro 3. Distribuição do tipo de intervenção, tempo de intervenção, tempo da sessão, frequência da intervenção e variáveis estudadas.

\begin{tabular}{|c|c|c|c|c|c|}
\hline ESTUDO & $\begin{array}{c}\text { TIPO DE } \\
\text { INTERVENÇĀO }\end{array}$ & $\begin{array}{l}\text { TEMPO DA } \\
\text { INTERVENÇĀO }\end{array}$ & $\begin{array}{c}\text { TEMPO } \\
\text { DA } \\
\text { SESSÃO }\end{array}$ & $\begin{array}{l}\text { FREQUÊNCIA DA } \\
\text { INTERVENÇÃO }\end{array}$ & VARIÁVEIS ESTUDADAS \\
\hline 1 & $\begin{array}{l}\text { Alongamento, } \\
\text { aquecimen-- } \\
\text { to, treino de } \\
\text { equilíbrio, } \\
\text { coordenação, } \\
\text { propriocep- } \\
\text { ção, desaque- } \\
\text { cimento. }\end{array}$ & 1 hora & 24 & $\begin{array}{c}3 \text { meses } 2 \text { vezes } \\
\text { por semana }\end{array}$ & $\begin{array}{c}\text { Sensibilidade, através do } \\
\text { monofilamento semmes- } \\
\text { weinstein } \\
\text { Funcionalidade, através } \\
\text { do teste timed up and go } \\
\text { (TUGG) } \\
\text { Dor, através da escala } \\
\text { visual analógica (EVA) } \\
\text { ADM, através da } \\
\text { goniometria } \\
\text { Força Muscular, escala } \\
\text { de força de Kendall. }\end{array}$ \\
\hline 2 & $\begin{array}{c}\text { Treino } \\
\text { proprioceptivo, } \\
\text { fortalecimento } \\
\text { muscular } \\
\text { com técnica } \\
\text { neuromuscular } \\
\text { proprioceptiva } \\
\text { (FNP), } \\
\text { deslizamento, } \\
\text { trabalho facial } \\
\text { e torcões } \\
\text { mecânica. }\end{array}$ & $60 \mathrm{~min}$. & 16 & $\begin{array}{c}2 \text { meses } 2 \text { vezes } \\
\text { por semana }\end{array}$ & \begin{tabular}{|} 
Equilíbrio postural, através \\
da plataforma de força \\
AMll OR6-6 2000 \\
Pressão plantar, através \\
do baropodômetro \\
eletrônico \\
Sensibilidade, através \\
do monofilamento \\
estesiômetro semmes- \\
weinstein \\
Dor, através da escala \\
visual analógica
\end{tabular} \\
\hline 3 & $\begin{array}{c}\text { Fortalecimento } \\
\text { do musculo } \\
\text { tibial anterior } \\
\text { no membro } \\
\text { comprometido } \\
\text { através } \\
\text { da analise } \\
\text { cronáxia, } \\
\text { tratamento } \\
\text { com o FES } \\
\text { associado } \\
\text { com exercício } \\
\text { de dorsiflexão } \\
\text { com } \\
\text { therabands } \\
\text { (verde, } \\
\text { vermelha e } \\
\text { dourada), } \\
\text { cinecioterapia } \\
\text { resistida com } \\
\text { theraband } \\
\text { dourada. }\end{array}$ & - & 4 & $\begin{array}{l}2 \text { semanas } \\
2 \text { vezes por } \\
\text { semana }\end{array}$ & $\begin{array}{c}\text { Força muscular, através } \\
\text { do Dinamômetro, } \\
\text { eletromiografo aparelho } \\
\text { nemesys. }\end{array}$ \\
\hline
\end{tabular}

Continua... 


\begin{tabular}{|c|c|c|c|c|c|}
\hline 4 & $\begin{array}{c}\text { Exercícios de } \\
\text { alongamento, } \\
\text { caminhada, } \\
\text { exercícios } \\
\text { do tipo ativo } \\
\text { livre e ativo } \\
\text { resistido, } \\
\text { massoterapia. }\end{array}$ & 1h e 30 min. & 08 & $\begin{array}{c}2 \text { meses } 1 \text { vez por } \\
\text { semana }\end{array}$ & $\begin{array}{l}\text { Sensibilidade, através } \\
\text { do Monofilamento de } \\
\text { Semmes-Weinstein. }\end{array}$ \\
\hline 5 & $\begin{array}{c}\text { Aquecimento } \\
\text { (movimento } \\
\text { ativo flexão } \\
\text { e extensão } \\
\text { do tornozelo, } \\
\text { movimento } \\
\text { circular do } \\
\text { tornozelo), } \\
\text { treinamento } \\
\text { proprioceptivo, } \\
\text { sensibilidade } \\
\text { ( lixa, escova } \\
\text { macia, toalha, } \\
\text { bola de massa- } \\
\text { gem, bola de } \\
\text { vidro), treino } \\
\text { de marcha e } \\
\text { equilíbrio, de- } \\
\text { saquecimento. } \\
\end{array}$ & - & 27 & $\begin{array}{c}2 \text { meses e } 7 \text { dias } \\
3 \text { vezes por } \\
\text { semana }\end{array}$ & $\begin{array}{c}\text { Qualidade de vida, } \\
\text { através do questionário } \\
\text { Short Form-36 Health } \\
\text { Survey (SF- 36) } \\
\text { Sensibilidade, através } \\
\text { de pincel, agulha, } \\
\text { álcool e monofilamento } \\
\text { estesiômetro semmes- } \\
\text { weintein } \\
\text { Pressão plantar, através } \\
\text { do baropodômetria } \\
\text { eletrônica } \\
\text { Funcionalidade, através } \\
\text { do timed up and Go } \\
\text { (TUG) }\end{array}$ \\
\hline 6 & $\begin{array}{c}\text { Conceito } \\
\text { balance, } \\
\text { atendimento } \\
\text { convencional } \\
\text { de fisioterapia } \\
\text { (estimulação } \\
\text { sensorial e } \\
\text { treino de } \\
\text { equilíbrio). }\end{array}$ & 1 hora & 12 & $\begin{array}{l}3 \text { vezes por } \\
\text { semana }\end{array}$ & $\begin{array}{l}\text { Equilíbrio, através do } \\
\text { teste de marcha Tandem } \\
\text { (TMT) e escala de } \\
\text { equilibrio de Berg (EEB) } \\
\text { Qualidade de vida, } \\
\text { através do questionário } \\
\text { Neuropathy- specific } \\
\text { quality of life (NeuroQol) } \\
\text { Sensibilidade, através do } \\
\text { monofilamento semmes- } \\
\text { weintein }\end{array}$ \\
\hline 7 & $\begin{array}{c}\text { Vibração } \\
\text { do corpo } \\
\text { inteiro (WBV) } \\
\text { combinada } \\
\text { com um } \\
\text { programa } \\
\text { de exercícios } \\
\text { de equilíbrio, } \\
\text { força muscular } \\
\text { e hemoglobina } \\
\text { glicosilada } \\
\text { (HbAlC). } \\
\end{array}$ & $60 \mathrm{~min}$. & 10 & $\begin{array}{l}1 \text { mês e } 14 \text { dias } \\
2 \text { vezes por } \\
\text { semana }\end{array}$ & $\begin{array}{c}\text { Equilíbrio, através da } \\
\text { Escala de equilíbrio de } \\
\text { Berg (EEB) } \\
\text { Funcionalidade, através } \\
\text { do teste up-and go } \\
\text { (TUG), teste de alcance } \\
\text { funcional (FRT). }\end{array}$ \\
\hline 8 & $\begin{array}{c}\text { Ganho } \\
\text { de ADM, } \\
\text { fortalecimento, } \\
\text { exercícios } \\
\text { funcionais, } \\
\text { treino de } \\
\text { caminhada. }\end{array}$ & 40 a 60 min. & 24 & $\begin{array}{l}3 \text { meses } 2 \text { vezes } \\
\text { por semana }\end{array}$ & $\begin{array}{l}\text { pressão plantar, através } \\
\text { do sistema Pedar-x } \\
\text { (novel, Munique, } \\
\text { Alemanha), utilizou o } \\
\text { software novel- projects. }\end{array}$ \\
\hline 9 & $\begin{array}{l}\text { Técnica de } \\
\text { frenquel, treino } \\
\text { de marcha, } \\
\text { equilíbrio e } \\
\text { propriocep- } \\
\text { ção, exercícios } \\
\text { respiratórios. }\end{array}$ & $45 \mathrm{~min}$. & 24 & $\begin{array}{c}3 \text { meses } 2 \text { vezes } \\
\text { por semana }\end{array}$ & $\begin{array}{c}\text { Pressão plantar, através } \\
\text { da } \\
\text { Sensibilidade, através do } \\
\text { monofilamento semmes- } \\
\text { weinstein. }\end{array}$ \\
\hline
\end{tabular}

Fonte: Dados da pesquisa, 2019. 


\section{DISCUSSÃO}

\section{ATUAÇÃO FISIOTERAPÊUTICA NA ALTERAÇÃO DE SENSIBILIDADE E DOR NA NEUROPATIA DIABÉTICA}

No estudo de Piovesan (2015) demonstrou também que houve diferença significativa na sensibilidade $(p<0,05)$ intragrupo e entre grupos. Já em questão a dor em membros inferiores, obteve diferença entre os grupos quando avaliada intervenção $(p=0,001)$ e intragrupo $G E(p=0,001)$.

Conforme estudo de Lima e Junqueira (2017) houve aumento na percepção plantar de 5,3\% para $23,4 \%$ de pontos sentidos com o monofilamento de cor azul $(0,02 \mathrm{~g})$ e diminuição de $30,3 \%$ para $13,6 \%$ pontos com monofilamento vermelho escuro $(4,0 \mathrm{~g})$, o que demonstra que houve melhora na sensibilidade do pé.

Conforme o estudo de Souza (2015) no que se refere aos resultados da sensibilidade testada com o Monofilamento Semmes-Weinstein em cada um dos 22 pontos das faces dorsal e plantar do pé direito e esquerdo (D1, D2, P1, P2, P3, P4, P5, P6, P7, P8 e P9), Em relação ao total de pontos plantares percebidos, foi realizada a comparação entre as pontuações pré e pós em cada grupo. No GC verificouse que 9 (42,8\%) dos casos apresentaram número de pontos mais elevados no pós, 7 (33,3\%) mantiverem o número de pontos inalterados e em 5 casos $(23,8 \%)$ o número de pontos sensíveis diminuiu. Mostrando que a fisioterapia contribuiu para a manutenção da sensibilidade tátil.

Segundo o estudo de Magno et al., (2017) foi demonstrado acréscimo médio positivo sobre a sensibilidade dos pontos analisados, resultando em melhora $(p=0,02)$. Neste caso outro estudo de Sales; Souza e Cardoso (2012) deixa-nos entender que a neuropatia periférica diabética é influência notável sistema sensorial cortical, portanto, táticas de reabilitação que tirem provisão visual, constituem no aumento da ativação e utilidade do controle sensorial periférico.

Conforme estudo de Santos et al., (2008) comparando que a sensibilidade antes, após seis e 12 semanas de treinamento proprioceptivo notou-se significativa melhora da sensibilidade tátil plantar $(p<0,05)$, apesar dos 12 pontos inicialmente estipulados, apenas $15 \%$ das voluntarias sentiam todos os pontos examinados. Após seis semanas de treino proprioceptivo, esse índice elevou-se para $46 \%$ e após 12 semanas, o índice atingiu $85 \%$.

\section{ATUAÇÃO FISIOTERAPÊUTICA NA ALTERAÇÃO DE ADM E FORÇA NA NÉUROPATIA DIABÉTICA}

Segundo Lima e Junqueira (2017) fizeram a comparação do grau de amplitude de movimento dos pacientes antes e depois da intervenção e observaram que houve diferença significativa para a goniometria de flexão plantar direita 
$(p=0,012)$ e esquerda $(p=0,019)$, onde foi observado aumento da ADM de ambas as articulações. Para flexão plantar do tornozelo direito houve um aumento médio de $24,4^{\circ}$ para $37,7^{\circ}$ e do lado esquerdo de $23,3^{\circ}$ para $34,9^{\circ}$ representando melhora de $50 \%$ e $54 \%$.

De acordo com o estudo de Coutinho et al., (2009) o grupo diabético membro intervenção, nas análises em média de reobase, acomodação e cronaxia variaram não apresentando grau de significância. Mas no fator de coeficiente alfa ocorreu grau de significância. Na análise dinamométrica do GDI e grupo hígido mostrou aumento em média da força muscular após intervenção em ambos, porém com grau de significância de 5\% para o GDI.

\section{ATUAÇÃO FISIOTERAPÊUTICA NA ALTERAÇÃO DE EQUILÍBRIO E PROPŘIOCEPÇÃO NA NEUROPATIA DIABÉTICA}

Conforme o estudo de Piovesan (2015) para ambos os pés foi encontrada diferença estatística na distribuição das cargas de pressão plantar intragrupo no Grupo Experimental, na região anterior, condições Semi-Tandem $(p=0,016)$, na região posterior para as duas condições também se observou diferença Romberg $(p=0,054)$ e ST $(p=0,016)$. Para os pés direito e esquerdo, foi encontrada diferença no GE na região do pé esquerdo, condição ST ( $p=0,001)$. Encontrou diferença entre os grupos pré- intervenção para a condição (R), na região médio- lateral direita $(p=0,055)$ e esquerda $(p=0,032)$. Em pés separados a diferença foi observada no pé esquerdo para ST no pós- teste região posterior $(p=0,41)$.

Segundo o estudo de Souza (2015) quanto avaliação de pressão plantar nas comparações intra-grupo, entre avaliação pré e pós, foi detectada diferença significativa na pressão média do pé direito no Grupo Controle $(p=0,041)$, indicando que a média no pós foi significativamente maior que no pré. No Grupo Intervenção esta diferença não foi significativa, pois a pressão média do pé direito se manteve com o estimulo de fisioterapia.

De acordo com o estudo de Magno et al., (2017) a estabilidade estática e dinâmica por meio das duas medidas de avaliação- TMT e EEB, foi demonstrado acréscimo médio positivo. Ressaltando que no GC apenas a EEB teve crescimento considerável $(p=0,03)$, enquanto no $G B$ todos os resultados obtiveram significância estatística $(p \leq 0,05)$, o que resultou melhora no equilíbrio analisado pela EEB $(p=0,04)$ e TMT $(p=0,03)$.

O estudo de Lee; Lee e Song (2013) observou que houve Melhorias significativas no equilíbrio estático, equilíbrio dinâmico, força muscular e HbAlc no grupo WBV, em comparação com os grupos BE e controle $(p<0,05)$. Assim, em combinação com o programa de exercícios de equilíbrio, a terapia de vibração do corpo inteiro (WBV), a curto prazo é benéfica na melhoria do equilíbrio, força 
muscular e HbAlc, em pacientes idosos com neuropatia diabética que estão em alto risco de sofrer quedas.

Segundo o estudo de Sartor et al., (2014) verificou que após o período de tratamento com o grupo intervenção houve um aumento no pico de pressão sob as áreas de seis pés e interação. Os efeitos foram encontrados no mediopé, curiosamente, reduções significativas aparecem no Gl após o período de seguimento, especialmente sob o mediopé lateral.

Conforme Santos et al., (2008) em relação aos valores médios de oscilação antero-posterior do centro de pressão, houve diferença significativa $(p<0,05)$ entre os valores ao longo do tempo, antes, após seis e 12 semanas de intervenção fisioterapêutica. Não houve diferença significativa $(p>0,05)$ quanto a oscilação médio-lateral entre o grupo.

\section{ATUAÇÃO FISIOTERAPÊUTICA NA QUALIDADE DE VIDA E FUNCIONALIDADE NA NEUROPATIA DIABÉTICA}

No estudo de Barros et al., (2017) observou que após a intervenção ocorreram alterações estatisticamente significantes em relação ao habito de andar sem calçados ( $p=0,003)$, a examinar os pés com frequência $(p=0,006)$, a não utilizar a pratica do escalda pés $(p=0,013)$, a secar corretamente os pés $(p=0,016)$. Com relação ao uso do calçado adequado e do emprego da massagem terapêutica nos pés, da hidratação adequada e da detecção e solução de eventuais alterações nos pés, a intervenção teve um impacto relevante $(p<0,001)$.

Segundo o estudo de Souza (2015) Em relação aos resultados do TUG, tanto na avaliação pré e pós, os dois grupos não diferiram estatisticamente. Após a intervenção, o Grupo Controle apresentou média de $26,2 \pm 26,1$ s, enquanto no grupo intervenção a média e DP foi de 19,5 $\pm 16,2 \mathrm{~s}(\mathrm{p}=0,377)$. Em questão no que diz respeito a qualidade de vida, Na avaliação final, após o período de intervenção / controle, o Gl apresentou escores significativamente mais altos que os do GC no Domínio Capacidade Funcional ( $p=0,013)$, no Domínio Aspecto Emocional $(p=0,007)$ e no Escore Geral 37 ( $p=0,022)$. Nos demais domínios (Limitações por Aspectos Físicos, Dor, Vitalidade, Aspectos Sociais e Saúde Mental) os grupos não diferiram estatisticamente após a intervenção.

Segundo estudo de Magno et al., (2017) em relação da qualidade de vida $(Q V)$, observou melhora significativa no índice geral do Neuroqol no período póstratamento no grupo balance (GB), assim como foram vislumbradas diferenças estatísticas nos domínios correspondentes aos sintomas sensentivo-motores $(p=0,03)$ e limitação das AVD's, sendo este último evidenciado nos $G B(p=0,04)$ e controle $(p=0,02)$. 


\section{CONCLUSÃO}

Este estudo permitiu avaliar o efeito da atuação fisioterapêutica na neuropatia diabética através de uma revisão integrativa, podendo observar a eficácia da atuação fisioterapêutica diante da neuropatia diabética.

O fisioterapeuta pode atuar desde a prevenção até a reabilitação do paciente acometido pela doença; com vários métodos e técnicas possíveis de estimular a sensibilidade, trabalhar força, equilíbrio e propriocepção, também pode fazer orientações quanto ao cuidado com os pés e hábitos de vida saudável.

No tocante a atuação do fisioterapeuta sobre a sensibilidade e dor alguns autores relataram em seus estudos que houve uma melhora significante em vários pontos de avaliação, mostrado que a intervenção fisioterapêutica sobre a sensibilidade e dor dos pacientes acometidos pela neuropatia diabética se faz necessário.

No que dizrespeito a intervenção do fisioterapeuta no ganho de ADM e força muscular, só dois artigos fizeram essa junção deixando claro que em seus estudos obtiveram um resultado significante quanto ao ganho de ADM e força muscular de membros inferiores. Em relação ao trabalho de equilíbrio e propriocepção alguns estudos revelam que houve melhora depois da intervenção fisioterapêutica e houve diferença significativa na qualidade de vida e funcionalidade dos pacientes. Tornado eficaz a atuação do fisioterapeuta na neuropatia diabética como mostra os resultados obtidos na pesquisa.

Vale ressaltar que ainda são poucos que conhecem sobre atuação fisioterapêutica na neuropatia diabética, fazendo-se necessárias novas pesquisas sobre o tema com uma população mais abrangente de pessoas com a neuropatia periférica diabética, além disso, a indisponibilidade de artigos na íntegra consiste em uma limitação do presente estudo.

\section{REFERÊNCIAS}

ANJOS, D. M. D. C. D. et al. Avaliação da capacidade funcional em idosos diabéticos. Fisioter. pesq., [s.l.], v. 19, n. 1, p. 73-80, 2012.

BARRILE, S. et al. Comprometimento sensório-motor dos membros inferiores em diabéticos do tipo 2. Fisioterapia em Movimento, Curitiba, v. 26, n. 3, p. 537-548, setembro 2013.

BARROS, M. D. F. A. et al. Impacto de intervenção fisioterapêutica na prevenção do pé diabético. Fisioterapia em Movimento, [s.I.], v. 25, n. 4, setembro 2017. 
BORGES, F. D. S.; CARDOSO, H. S. G. Avaliação sensório-motora do tornozelo e pé entre idosos diabéticos e não diabéticos. Revista Bras. Geriatr. Gerontol., Rio de Janeiro, v. 13, n. 1, p. 93-102, 2010.

CARDOSO, L. M. et al. Aspectos importantes na prescrição do exercício fisico para o diabetes mellitus tipo 2. Revista brasileira de prescrição e fisiologia do exercício, São Paulo, v. 1, n. 6, p. 59-69, novembro/dezembro 2011.

CARVALHO, V. F. D. et al. Limiar de sensibilidade cutânea dos pés em pacientes diabéticos através do pressure specified sensory device: uma avaliação da neuropatia. Revista da associação médica brasileira, [s.l.], v. 55, n. 1, p. 29-34, 2009.

CENCI, D. R. et al. Analise do equilíbrio em pacientes diabéticos por meio do sistema F-Scan e da escala de equilíbrio de Berg. Fisioterapia em Movimento, Curitiba, v. 26, n. 1, p. 55-61, janeiro/março 2013.

CISNEROS, L. L. Avaliação de um programa para prevenção de úlceras neuropáticas em portadores de diabetes. Revista Brasileira de Fisioterapia, São Carlos, v. 14, n. 1, p. 31-37, fevereiro 2010.

COUTINHO, F. M.; SOARES, M. A.; SILVA, J. D. intervenção fisioterapeutica agudo em idosas portadoras de diabetes mellitus controlada por analise dinamometrica e cronaxia. perspectivasonline 2007-2011, 2009.

CUBAS, M. R. et al. Pé diabético: orientações e conheciento sobre cuidado preventivo. fisioter. mov., v. 26, n. 3, p. 647-655, jul/set 2013.

DANTAS, I. F. et al. Intervenção da fisioterapia na neuropatia. Eventos.set.edu, 2014. Disponivel em: <https://eventos.set.edu.br/index.php/sempesq/article/view/423/0>. Acesso em: 15 março 2018.

FERREIRA, L. T. et al. Diabetes mellitus: hiperglicemia crônica e suas complicações. Arquivos brasileiros de ciências da saúde, [s.I.], v. 36, n. 3, p. 182-188, setembro/ dezembro 2011.

FLOR, L. S.; CAMPOS, M. R. Prevalência de diabetes mellitus e fatores associados na população adulta brasileira: evidências de um inquérito de base populacional. Revista brasileira de epidemiologia, [s.I.], v. 20, n. 1, p. 16-29, janeiro/março 2017.

FORTALEZA, A. C. D. S. et al. Avaliação clínica da sensibilidade em indivíduos com diabetes melito. colloquium vital, v. 2, n. 2, p. 44-49, jul/dez 2010. 
FORTE, F. J. et al. Efeitos do tratamento fisioterapeutico na neuropatia periférica causada pelo diabetes: um relato de caso. Encontro Universitario da UFC, Fortaleza, v. 1, n. 1, p. 3775, 2016.

FREGONESI, E. T.; CAMARGO, M. R. D. Parâmetros da marcha em portadores de diabetes mellitus. Revista brasileira cineantropom. desenpenho humano, Florianópolis, v. 12, n. 2, p. 155-163, abril 2010.

FURIERI, F. P. M. atuação fisioterapeutica com ultrasson em pacientes com neuropatia periferica diabetica., 2015.

KRAYCHETE, D. C.; SOKATA, R. K. Neuropatias periféricas dolorosas. Revista Bras. Anestesiol., Campinas, v. 61, n. 5, p. 649-658, outubro 2011.

LEE, K.; LEE, S.; SONG, C. whole-body vibration training improves balance, muscle strength and blycosylated hemoglobin in elderly patients with diabetic neuropathy. journal of experimental medicine, v. 231, n. 4, p. 305-314, 2013.

LIMA, C. F. D.; JUNQUEIRA, K. B. Efeitos de uma intervenção fisioterapeutica de equilibrio, propriocepção e coordenação em diabéticos. Universidade de Brasília, Faculdade de Ceilândia. Brasília, p. 45. 2017.

MAGNO, L. D. et al. Fisioterapia convencional versus conceito balance sobre alterações sensorio-motoras da neuropatia diabética. reserch medical journal, v. 1, n. 1, p. 0-0, 2017.

MANTOVANI, A. M. et al. Estudo comparativo das representações sociais sobre diabetes mellitus e pé diabético. Cad. saúde publica, Rio de Janeiro, v. 29, n. 12, p. 2427-2435, dezembro 2013.

MENDES, T. D. A. B. et al. Diabetes mellitus: fatores associados à prevalência em idosos, medidas e práticas de controle e uso dos serviços de saúde em São Paulo, Brasil. Cad. Saúde Publica, Rio de Janeiro, v. 27, n. 6, p. 1233-1243, junho 2011.

MENDONÇA, S. D. S.; MORAIS, J. D. S.; MOURA, M. C. G. G. D. Proposta de um protocolo de avaliação fisioterapêutica para os pés diabéticos. Fisioterapia em Movimento, Curitiba, v. 24, n. 2, p. 285-298, abril/junho 2017.

NOZABIELI, A. J. et al. Analise do equilíbrio postural de indivíduos diabéticos por meio de baropodometria. Revista motricidade, [s.I.], v. 8, n. 3, p. 30-39, 2012. 
OLIVEIRA, A. F. D.; MARCHI, C. B. D.; LEGUISAMO, C. P. Diabetic footwear: is it an assistive technology capable of reducing peak plantar pressures in elderly patients with neuropathy? Fisioterapia em Movimento, Curitiba, v. 29, n. 3, p. 469-476, sept 2016.

PASQUALOTO, R.; ALBERTON, D.; FRIGERI, H. R. Diabetes e complicações. Jornal of Biotechnalogyand Biodiversity, [S.I.], v. 3, n. 4, dezembro 2012.

PEDROSA, H. Neuropatia diabética periférica. In: sociedade brasileira de diabetes (SBD), 2009. Disponivel em: <https://www.diabetese.book.org.br/capitulo/ neuropatia_diabética_periférica>. Acesso em: 15 março 2018.

PETERMANN, X. B. et al. Epidemiologia e cuidados à diabetes mellitus praticado na atenção primaria a saúde: uma revisão narrativa. Revista Saúde (Santa Maria), Santa Maria-RS, v. 41, n. 1, p. 49-56, janeiro/julho 2015.

PINCON, A. P. Estudo da progressão da diabetes e da neuropatia periferica: classificação da severidade e caracterização cinética da locomoção. Faculdade de medicina, university of São Paulo. São Paulo, p. 103. 2011.

PIOVESAN, A. C. Efeitos de um tratamento fisioterapeutico na dor, equilibrio postural, distribuição das cargas de pressão plantar e sensibilidade de idosas diabéticas tipo 2. Universidade Federal de Santa Maria. RS, p. 94. 2015.

PIRES, S. R.; SANDOVAL, R. A. Perfil de diabéticos amputados de membro inferior atendido no serviço de fisioterapia do centro de reabilitação e readaptação Dr. Henrique Santillo Crer. Trances, [s.I.], v. 2, n. 4, p. 213-224, 2010.

PORTES, L. H. Abordagem do fisioterapeuta no diabetes mellitus: revisão de literatura. Arq. Ciênc. Saúde, [s.l.], v. 22, n. 3, p. 9-14, julho/setembro 2015.

SALES, K. L. D. S.; SOUZA, L. A. D.; CARDOSO, V. S. Equilíbrio estático de indivíduos com neuropatia periférica diabética. Fisioterapia e Pesquisa [S.I.], v. 19, n. 2, p. 122-127, junho 2012.

SANTOS, A. A. et al. efeitos do treinamento proprioceptivo em mulheres diabéticas. revista brasileira de fisioterapia, v. 12, n. 3, p. 183-187, 2008.

SANTOS, M. D. L. D. et al. Comparação dos valores do índice tornozelo-braço entre idosos diabéticos e não diabéticos. Revista Humano Ser- UNIFACEX, Natal-RN, v. 1, n. 1, p. 18-31, 2015. 
SARTOR, C. D. Influência da intervenção cinesioterapêutica em tornozelo e pé na biomecânica da marcha de diabéticos neuropatas: um ensaio clínico radomizado. Faculdade de medicina, university of São Paulo. São Paulo. 2013.

SARTOR, C. D. et al. effects of strengthening, streteching and funcional training on foot fuction in patients with diabetic neuropathy: resutts of a radomized trial. BMC musculoskeletal disorders, v. 15, n. 1, p. 137, 2014.

SHIWA, S. R. et al. Reprodutibilidade da Versão em Português de Escala de PEDrO. caderneta saúde pública, v. 27, n. 10, p. 2063-2068, outubro 2011.

SILVA, A. G. D.; RODRIGUES, L. C.; HONÓRIO, G. J. D. S. Analise do equilíbrio bipodal em diabéticos com neuropatia periférica. Revista Fisioter. Bras., [s.I.], v. 10, n. 4, p. 248-251, julho/agosto 2009.

SILVA, C. A. M. D. et al. Pé diabético e avaliação do risco de ulceração. revista de enfermagem referência, V. IV, n. 1, fev/mar 2014.

SILVA, J. V. D. et al. Assistência e acompanhamento de enfermagem a mulheres com diabetes gestacional. Nursing, São Paulo, v. 20, n. 226, p. 1632-1635, março 2017.

SILVA, L. A. L. D. Avaliação de diabéticos antes e após a inserção de um programa de exercício físico. Trabalho de Conclusão de Curso ( Bacharelado em Fisioterapia)Universidade de Brasília. Brasília, p. 39. 2015.

SILVA, V. C. D. et al. Fisioterapia e neuropatia periférica em portadores de diabetes melito II: produção bibliografica entre 1966 e 2011 . Revista fisioterapia s-fun, Fortaleza, v. 1, n. 1, p. 47-51, janeiro/junho 2012.

SOUZA, C. C. D. Eficácia de uma intervenção fisioterapeutica na qualidade de vida, propriocepção, sensibilidade e mobilidade de idosos diabéticos institucionalizados. Universidade Católica do Rio Grande do Sul. porto alegre, p. 89. 2015.

SOUZA, J. M. et al. Conhecimentos e atitudes dos acadêmicos concludentes de fisioterapia quanto aos cuidados preventivos no pé diabético. Revista interdisciplinar, [s.l], v. 6, n. 4, p. 124-131, outubro/dezembro 2013.

TESTON, E. F. et al. Fatores associados ao conhecimento e à atitude em relação ao diabetes mellitus. Cogitare enferm., [s.I.], v. 22, n. 4, p. 1-10, outubro/dezembro 2017. ULHOA, L. S. et al. Mobilidade articular de idosos diabéticos e não diabéticos e influência da fisioterapia. Fisioterapia em Movimento, Curitiba, v. 24, n. 1, p. 99-106, Janeiro/Março 2011. 
VERAS, T. C. et al. Associação entre força muscular e sensibilidade plantar em pacientes diabéticos: um estudo transversal. saude e pesquisa, s.l., v. 8, n. 3, p. 525532, 2015. 